\title{
Two Server (s, S) Inventory System with Positive Service Time, Positive Lead Time, Retrial Customers and Negative Arrivals
}

\author{
Hannah Revathy.P \\ Bharathiar University \\ Coimbatore \\ India
}

\author{
Muthu Ganapathi \\ Subramanian \\ Tagore Arts College \\ Pondicherry \\ India
}

\begin{abstract}
This paper deals with a two server $(\mathrm{s}, \mathrm{S})$ inventory system with positive service time, positive lead time, retrial of customers and negative arrivals. In this system, arrival of customers form a Poisson process, lead time and service time are exponentially distributed. The system starts with $\mathrm{S}$ units of inventory on hand. Each arriving customer is served a single unit of the item by any one of the servers. When the inventory level reaches s, an order is placed for (S-s) units. If the inventory level is zero or both servers are busy, then the arriving customer goes to orbit and becomes a source of repeated calls. Assume that the capacity of the orbit is infinite. The negative arrival plays an important role in this paper and it controls the congestion in the orbit by removing one customer from the orbit and further it is assumed that it removes the customer from the orbit only if inventory level is zero or both servers are busy. It is also assumed that the access from orbit to the service facility is governed by the classical retrial policy. This model is solved by using Direct Truncation Method. Numerical and graphical studies have been done for analysis of mean number of customers in the orbit, average inventory level, Truncation level, mean number of busy servers and system performance measures. A suitable cost function is defined.
\end{abstract}

\section{General Terms}

Stochastic Process, Queueing theory, Retrial queues,

Inventory system, Negative arrivals.

\section{Keywords}

$(\mathrm{s}, \mathrm{S})$ inventory system, positive service time, Positive lead time, retrial customers, LDQBD Process, negative arrivals

\section{INTRODUCTION}

Many researchers have studied inventory systems for decades but only recently their attention has turned towards retrial customers in inventory system. These models consider situations in which the arriving customers who find all servers busy or the inventory level zero may retry for service after sometime. A .Krishnamoorthy and Islam [9] have studied inventory system with postponed demands. J. R. Artalejo and A. Krishnamoorthy [8] have studied $(\mathrm{s}, \mathrm{S})$ inventory systems with repeated attempts and P.V. Ushakumari [13] considering random lead time. A .Krishnamoorthy and K.P.Jose [10] have studied the system with positive lead time, loss and retrial of customers. Gelenbe [5], Harrison and Pitel [6], Artalejo and Gomez-Corral [2] who have studied the negative arrivals which removes a customer or a batch of customers from the system according to some strategy.
This paper is organized as follows. Section 2 gives the model description and analysis while section 3 highlights the direct truncation method used. Section 4 discusses the stability condition and the performance measures and section 5 provides the cost analysis and numerical results followed by conclusion as section 6 .

\section{MODEL DESCRIPTION}

In this paper, a $(s, S)$ inventory retrial queueing system in which arrival follows a Poisson process with parameter $\lambda$ is considered. Lead time and service time are exponentially distributed with parameter $v$ and $\mu$ respectively. The negative arrival rate follows a Poisson distribution with parameter $\lambda_{-1}$. The system starts with S units of inventory on hand. Each arriving customer is served a single unit of the item by any one of the servers. When the inventory level reaches s, an order is placed for (S-s) units. If the inventory level is zero or both servers are busy, then the arriving customer goes to orbit and becomes a source of repeated calls. Assume that the capacity of the orbit is infinite. The negative arrival plays an important role in this paper and it controls the congestion in the orbit by removing one customer from the orbit and further it is assumed that it removes the customer from the orbit only if inventory level is zero or both servers are busy.

Most of the queueing system with repeated attempts assume that each customer in the retrial group seeks service independently of each other after a random time exponentially distributed with rate $\sigma$ so that the probability of repeated attempt during the interval $(t, t+\Delta \mathrm{t})$ given that there were $\mathrm{n}$ customers in orbit at time $t$ is $n \sigma \Delta \mathrm{t}+\mathrm{O}(\Delta \mathrm{t})$. This discipline of access to the server from the retrial group is called classical retrial policy. The input flow of primary calls, interval between repeated calls, negative arrival and service time are mutually independent.

Let $N(t)$ be the random variable which represents the number of customers in orbit at time $\mathrm{t}, \mathrm{B}(\mathrm{t})$ be the number of busy servers at time $\mathrm{t}$ and $\mathrm{I}(\mathrm{t})$ be the random variable which represents the inventory level at time $t$.

The random process is described as

$\{\langle\mathrm{N}(\mathrm{t}), \mathrm{B}(\mathrm{t}), \mathrm{I}(\mathrm{t})\rangle / \mathrm{N}(\mathrm{t})=0,1,2,3, \ldots ; \mathrm{B}(\mathrm{t})=0,1,2$

$\mathrm{I}(\mathrm{t})=0,1,2,3, \ldots \mathrm{s}, \mathrm{s}+1, \ldots \mathrm{S}\}$

The possible state space is

$\{(i, 0, j), i \geq 0,0 \leq j \leq S\} U\{(i, 1, j), i \geq 0,1 \leq j \leq S\} U$ 
$\{(i, 2, j), i \geq 0,2 \leq j \leq S\}$

The infinitesimal generator matrix $\mathbf{Q}$ is a block tri diagonal matrix given below

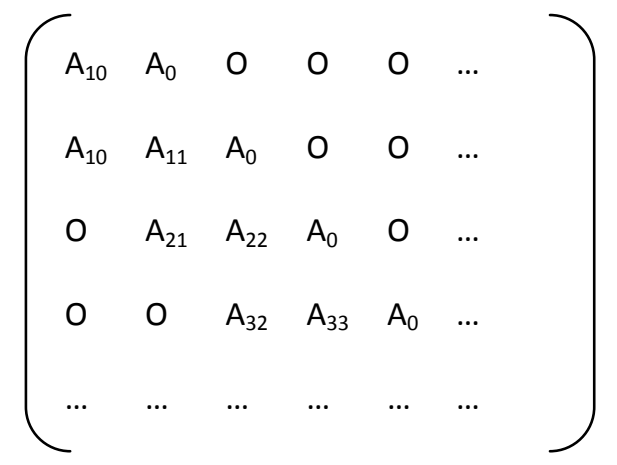

Where the blocks $A_{0}, A_{1, i}(i \geq 0)$, and $A_{2, i}(i \geq 1)$, are square matrices of order $3 \mathrm{~S}$

they are given by

$$
\begin{aligned}
& A_{0}=\left(\begin{array}{ccc}
B_{0} & 0 & 0 \\
0 & 0 & 0 \\
0 & 0 & B_{1}
\end{array}\right) \\
& A_{1,1}=\left(\begin{array}{lll}
E_{0} & E_{1} & 0 \\
E_{2} & E_{10} & E_{4} \\
0 & E_{5} & E_{0}
\end{array}\right) \quad A_{2 i}=\left(\begin{array}{ccc}
C_{0} & C_{1} & 0 \\
0 & 0 & C_{2} \\
0 & 0 & C_{7}
\end{array}\right)
\end{aligned}
$$

Where

$$
\begin{aligned}
& B_{0}=\left(\begin{array}{cccc}
\lambda & 0 & \cdots & 0 \\
0 & 0 & \cdots & 0 \\
\vdots & \vdots & \cdots & \vdots \\
0 & 0 & \cdots & 0 \\
0 & & &
\end{array}\right)_{(s+1) \times[S+1)]} \\
& B_{1}=\left(\begin{array}{cccc}
\lambda & 0 & \cdots \infty & 0 \\
0 & \lambda & \cdots & 0 \\
\vdots & \vdots & \cdots & \vdots \\
0 & 0 & \cdots & \lambda
\end{array}\right)_{(s-1) \times(s-1)}
\end{aligned}
$$

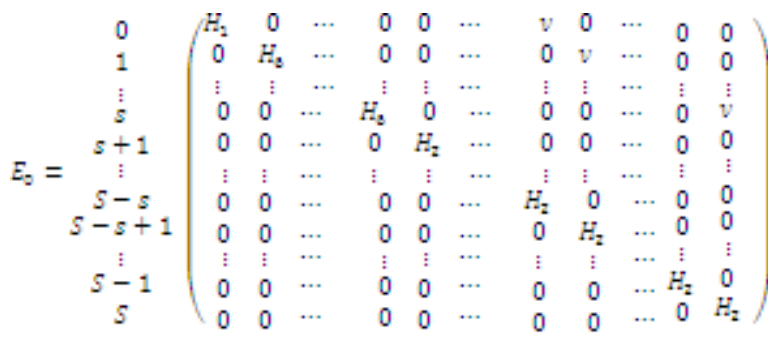

$$
\begin{aligned}
& E_{1}=\left(\begin{array}{cccc}
0 & 0 & \cdots & 0 \\
\lambda & 0 & \cdots & 0 \\
0 & \lambda & \cdots & 0 \\
\vdots & \vdots & \cdots & \vdots \\
0 & 0 & \cdots & \lambda
\end{array}\right)
\end{aligned}
$$$$
E_{2}=\left(\begin{array}{cccc}
\mu & 0 & x-\infty & 0 \\
0 & \mu & \cdots \infty & 0 \\
\vdots & \vdots & \cdots & \vdots \\
0 & 0 & \cdots \infty & \mu \\
0 & & &
\end{array}\right)_{S N(S+1)}
$$

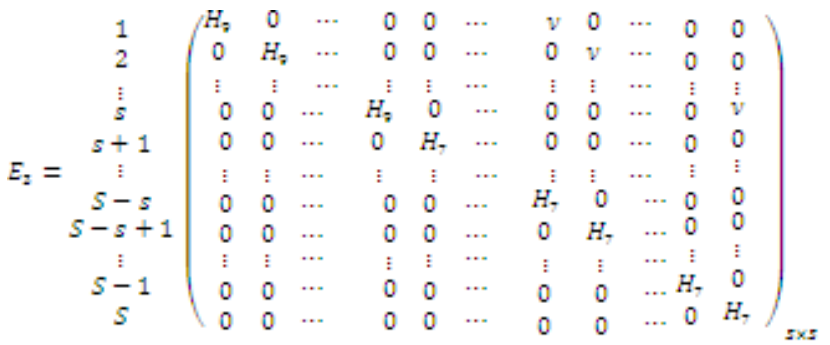$$
E_{4}=\left(\begin{array}{cccc}
0 & 0 & \cdots \infty & 0 \\
\lambda & 0 & \cdots & 0 \\
0 & \lambda & \cdots & 0 \\
\vdots & \vdots & \cdots & \vdots \\
0 & 0 & \cdots & \lambda
\end{array}\right)_{5 \times(5-1)}
$$

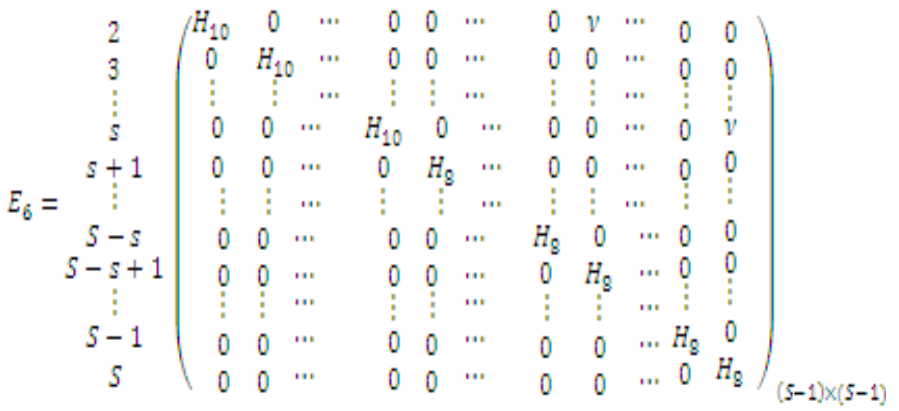$$
C_{0}=\left(\begin{array}{cccc}
\lambda_{-1} & 0 & \cdots & 0 \\
0 & 0 & \cdots & 0 \\
\vdots & \vdots & \cdots & \vdots \\
0 & 0 & \cdots & 0
\end{array}\right)_{(S+1) \times[S+1)}
$$$$
C_{1}=\left(\begin{array}{cccc}
0 & 0 & \cdots & 0 \\
i \sigma & 0 & \cdots & 0 \\
0 & i \sigma & \cdots & 0 \\
\vdots & \vdots & \cdots & \vdots \\
0 & 0 & \cdots & i \sigma
\end{array}\right)
$$$$
C_{2}=\left(\begin{array}{cccc}
0 & 0 & \cdots & 0 \\
i \sigma & 0 & \cdots & 0 \\
0 & i \sigma & \cdots & 0 \\
\vdots & \vdots & \cdots & \vdots \\
0 & 0 & \cdots & i \sigma
\end{array}\right)_{S \sin (S-1]}
$$$$
C_{9}=\left(\begin{array}{cccc}
\lambda_{-1} & 0 & \cdots \infty & 0 \\
0 & \lambda_{-1} & \cdots & 0 \\
\vdots & \vdots & \cdots \infty & \vdots \\
0 & 0 & \cdots & \lambda_{-1}
\end{array}\right)_{(5-1) \times[S-1]}
$$ 


\section{DESCRIPTION OF COMPUTATIONAL METHOD}

Retrial queueing models can be solved computationally by the various techniques. One of the feasible techniques is Direct Truncation Method. This method is applied in this paper for computational purpose for finding the Steady state probability vector and described as below

\section{DIRECT TRUNCATION METHOD}

Let $\mathbf{X}$ be the steady-state probability vector of $\mathbf{Q}$, partitioned as $\mathbf{X}=(\mathbf{x}(\mathbf{0}), \mathbf{x}(\mathbf{1}), \mathbf{x}(\mathbf{2}), \ldots)$ where $\mathbf{X}$ satisfies

$$
\mathbf{X Q}=\mathbf{0} \text { and } \mathbf{X e}=\mathbf{1}
$$

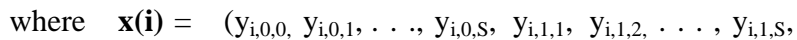
$\left.\mathrm{y}_{\mathrm{i}, 2,2}, \mathrm{y}_{\mathrm{i}, 2,3}, \ldots, \mathrm{y}_{\mathrm{i}, 2, \mathrm{~S}}\right) ; \mathrm{i}=0,1,2, \ldots$

The above system of equations can be solved by means of truncating the system of equations for sufficiently large value of the number of customers in the orbit, say M. That is, the orbit size is restricted to $\mathrm{M}$ such that any arriving customer finding the orbit full is considered lost. The value of $\mathrm{M}$ can be chosen so that the loss probability is small. Due to the intrinsic nature of the system, the only choice available for studying $\mathrm{M}$ is through algorithmic methods. While a number of approaches are available for determining the cut-off point, $\mathrm{M}$, the one that seems to perform well is to increase $\mathrm{M}$ until the largest individual change in the elements of $\mathbf{X}$ for successive values is less than $\varepsilon$ a predetermined infinitesimal value.

If $\mathrm{M}$ denotes the cut-off point or Truncation level, then the steady state probability vector $\mathbf{X}^{(\mathbf{M})}$ is partitioned as $\mathbf{X}^{(\mathbf{M})}=$ $(\mathbf{x}(\mathbf{0}), \mathbf{x}(\mathbf{1}), \mathbf{x}(\mathbf{2}), \ldots, \mathbf{x}(\mathbf{M}))$, where $\mathbf{X}^{(\mathbf{M})}$ satisfies

$$
\mathbf{X}^{(\mathbf{M})} \mathbf{Q}^{(\mathbf{M})}=\mathbf{0} \text { and } \mathbf{X}^{(\mathbf{M})} \mathbf{e}=1,
$$

where $x(i)=\left(y_{i, 0,0}, y_{i, 0,1}, \ldots, y_{i, 0, S}, y_{i, 1,1}, y_{i, 1,2}, \ldots, y_{i, 1, S}, y_{i, 2,2}\right.$, $\left.\mathrm{y}_{\mathrm{i}, 2,3}, \ldots, \mathrm{y}_{\mathrm{i}, 2, \mathrm{~S}}\right) ; \mathrm{i}=0,1,2, \ldots, \mathrm{M}$.

$Q=\left(\begin{array}{ccccccccc}A_{1,0} & A_{0} & 0 & 0 & 0 & 0 & \cdots & 0 & 0 \\ A_{21} & A_{1,1} & A_{0} & 0 & 0 & 0 & \cdots & 0 & 0 \\ 0 & A_{2,2} & A_{1,2} & A_{0} & 0 & 0 & \cdots & 0 & 0 \\ \vdots & \vdots & \vdots & & \vdots & \vdots & & \vdots & \vdots \\ 0 & 0 & 0 & 0 & 0 & 0 & \cdots & A_{2 N} & A_{1, N}\end{array}\right)$

The above system of equations is solved exploiting the special structure of the co-efficient matrix. It is solved using Numerical methods. Since there is no clear cut choice for M, the iterative process may be started by taking, say $M=1$ and increase it until the individual elements of $\mathbf{X}$ do not change significantly. That is, if $\mathrm{M}^{*}$ denotes the truncation point then

$$
\left\|\mathbf{x}^{\mathbf{M}^{*}}(\mathbf{i})-\mathbf{x}^{\mathbf{M}^{*-1}}(\mathbf{i})\right\|_{\infty}<\varepsilon
$$

where $\boldsymbol{\varepsilon}$ is an infinitesimal quantity.

\section{STABILITY ANALYSIS}

Let $\pi$ denote the steady state probability vector of the generator $A=A_{0}+A_{1}+A_{2}$. The vector $\pi$ partitioned as $\pi=\left(\pi_{0}, \pi_{1}, \ldots \pi_{\mathrm{s}}, \ldots . \pi_{3 \mathrm{~S}-1}\right)$ can be got by solving $\pi \mathrm{A}=0$ and $\pi \mathrm{e}=1$. The steady state probability vector $X$ exists if and only if the condition $\pi \mathrm{A}_{0} \mathrm{e}<\pi \mathrm{A}_{2} \mathrm{e}$

holds good. This reduces to $\lambda\left[\pi_{0}+\sum_{j=25+1}^{\infty} \pi_{j}\right]<M \sigma\left[\sum_{j=1}^{\infty} \pi_{j}+\sum_{j=5+2}^{\infty} \pi_{j}\right]+\lambda_{-1}\left[\left[\pi_{0}+\sum_{j=15+1}^{\infty} \pi_{j}\right]\right.$

which gives the required stability condition for this model.

\section{SYSTEM PERFORMANCE MEASURES}

The expected inventory level is given by

$$
E I L=\sum_{i=0}^{\infty} \sum_{j=0}^{S} j y_{i, 0, j}+\sum_{i=0}^{\infty} \sum_{j=1}^{S} j y_{i, 1, j}+\sum_{i=0}^{\infty} \sum_{j=2}^{S} j y_{i, 2, j}
$$

The expected number of customers in the orbit is given by

$$
E N C O=\sum_{i=0}^{\infty} i\left(\sum_{j=0}^{S} y_{i, 0, j}+\sum_{j=1}^{S} y_{i, 1, j}+\sum_{j=2}^{S} y_{i, 2, j}\right)
$$

The expected reorder rate is given by

$$
\boldsymbol{E} \boldsymbol{R} \boldsymbol{O R}=\boldsymbol{\mu} \sum_{i=0}^{\infty} y_{i, 1, s+1}+2 \mu \sum_{j=2}^{\infty} y_{i, 2, j}
$$

The expected number of departures after completing service (ENDS) is given by

$$
E N D S=\mu \sum_{I=0}^{\infty} \sum_{j=1}^{S} y_{i, 1, j}+2 \mu \sum_{I=0}^{\infty} \sum_{j=1}^{S} y_{i, 2, j}
$$

\section{COST ANALYSIS}

Different costs are defined as

$\mathrm{K}=$ fixed cost,

$\mathrm{C}_{1}=$ procurement cost/unit,

$\mathrm{C}_{2}=$ holding cost of inventory/unit /unit time,

$\mathrm{C}_{3}=$ holding cost of customers/unit /unit time,

$\mathrm{C}_{4}=$ cost due to service/unit /unit time,

$\mathrm{C}_{5}=$ revenue from service/unit/unit time.

A cost function defined as the expected total cost (ETC) of the system is introduced, which is given by

$$
\begin{aligned}
& \qquad \begin{array}{c}
\mathrm{ETC}=\left[\mathrm{K}+(\mathrm{S}-\mathrm{s}) \mathrm{C}_{1}\right] \mathrm{EROR}+\mathrm{C}_{2} \mathrm{EIL}+\mathrm{C}_{3} \mathrm{ENCO}+ \\
\left(\mathrm{C}_{5}-\mathrm{C}_{4}\right) \text { ENDS }
\end{array} \\
& \text { Numerical Examples }
\end{aligned}
$$

This section gives the numerical results of this model for various values of the parameters $\lambda, \lambda_{-1}, \mu, v, \sigma, s$ and $S$ chosen in such a way that they satisfy the stability conditions given in section 4.

System performance measures like Expected Number of Customers in the Orbit (ENCO) and Expected Inventory Level (EIL ) are found using the steady state probability vector $\mathrm{X}$ for different values of $\nu$ and $\sigma$ fixing $\lambda, \mu, \mathrm{s}$ and $\mathrm{S}$ and some are tabulated below. 
Table 5.1 Variations in Retrial rate for $\lambda=10 \mu=20 \quad,=10 \quad s=3 \quad S=6 \quad \lambda=6$

\begin{tabular}{|c|c|c|c|}
\hline$\sigma$ & $\mathrm{M}$ & ENCO & EIL \\
\hline 10 & 17 & 0.2013 & 3.9616 \\
\hline 20 & 17 & 0.1457 & 3.9544 \\
\hline 30 & 17 & 0.1264 & 3.951 \\
\hline 40 & 17 & 0.1164 & 3.949 \\
\hline 50 & 17 & 0.1104 & 3.9477 \\
\hline 60 & 17 & 0.1063 & 3.9467 \\
\hline 70 & 17 & 0.1033 & 3.946 \\
\hline 80 & 17 & 0.1011 & 3.9455 \\
\hline 90 & 17 & 0.0993 & 3.9451 \\
\hline 100 & 17 & 0.0979 & 3.9447 \\
\hline 200 & 17 & 0.0915 & 3.9431 \\
\hline 300 & 17 & 0.0893 & 3.9425 \\
\hline 400 & 17 & 0.0882 & 3.9423 \\
\hline 500 & 17 & 0.0875 & 3.9421 \\
\hline 600 & 17 & 0.0871 & 3.942 \\
\hline 700 & 17 & 0.0868 & 3.9419 \\
\hline 800 & 17 & 0.0865 & 3.9418 \\
\hline 900 & 17 & 0.0863 & 3.9418 \\
\hline 1000 & 17 & 0.0862 & 3.9417 \\
\hline 2000 & 17 & 0.0855 & 3.9416 \\
\hline 3000 & 17 & 0.0853 & 3.9415 \\
\hline 4000 & 17 & 0.0852 & 3.9415 \\
\hline 5000 & 17 & 0.0851 & 3.9415 \\
\hline 6000 & 17 & 0.0851 & 3.9414 \\
\hline 7000 & 17 & 0.085 & 3.9414 \\
\hline 8000 & 17 & 0.085 & 3.9414 \\
\hline 9000 & 17 & 0.085 & 3.9414 \\
\hline & & & \\
\hline
\end{tabular}

Table 5.2 Variations in Retrial rate for $\lambda=10 \quad \mu=20 \quad i=20 \quad s=3 \quad \mathrm{~S}=6 \quad \lambda=6$

\begin{tabular}{|c|c|c|c|}
\hline$\sigma$ & $\mathrm{M}$ & ENCO & EIL \\
\hline 10 & 11 & 0.1189 & 4.5003 \\
\hline 20 & 11 & 0.0778 & 4.4985 \\
\hline 30 & 11 & 0.0635 & 4.4976 \\
\hline 40 & 11 & 0.0562 & 4.497 \\
\hline 50 & 11 & 0.0517 & 4.4967 \\
\hline 60 & 11 & 0.0487 & 4.4964 \\
\hline 70 & 11 & 0.0466 & 4.4962 \\
\hline 80 & 11 & 0.0449 & 4.4961 \\
\hline 90 & 11 & 0.0436 & 4.4959 \\
\hline 100 & 11 & 0.0426 & 4.4958 \\
\hline 200 & 11 & 0.0379 & 4.4954 \\
\hline 300 & 11 & 0.0362 & 4.4952 \\
\hline 400 & 11 & 0.0354 & 4.4951 \\
\hline 500 & 11 & 0.0349 & 4.4951 \\
\hline 600 & 11 & 0.0346 & 4.495 \\
\hline 700 & 11 & 0.0344 & 4.495 \\
\hline 800 & 11 & 0.0342 & 4.495 \\
\hline 900 & 11 & 0.0341 & 4.495 \\
\hline 1000 & 11 & 0.0339 & 4.495 \\
\hline 2000 & 11 & 0.0334 & 4.4949 \\
\hline 3000 & 11 & 0.0333 & 4.4949 \\
\hline 4000 & 11 & 0.0332 & 4.4949 \\
\hline 5000 & 11 & 0.0331 & 4.4949 \\
\hline 6000 & 11 & 0.0331 & 4.4949 \\
\hline 7000 & 11 & 0.0331 & 4.4949 \\
\hline 8000 & 11 & 0.0331 & 4.4949 \\
\hline 9000 & 11 & 0.033 & 4.4949 \\
\hline
\end{tabular}

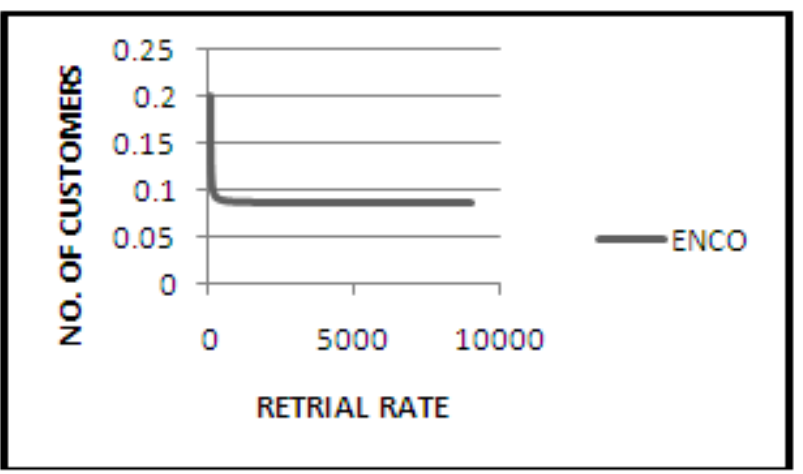

Figure 5.1 Expected Number of Customers in the Orbit When $\lambda=10 \mu=20,=10_{s}=3 \quad S=6 \quad \lambda_{-1}=6$

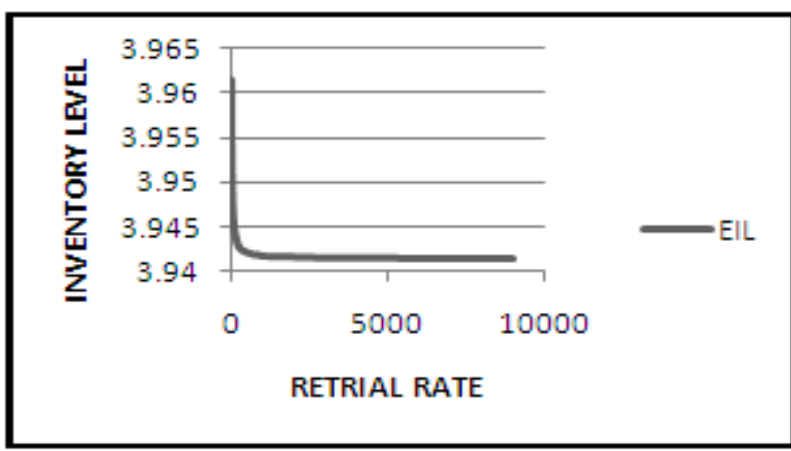

Figure 5,2 Expected Inventory Level

When $\lambda=10 \mu=20 \quad v=10 \quad s=3 \quad \mathrm{~S}=6 \quad \lambda_{-1}=6$

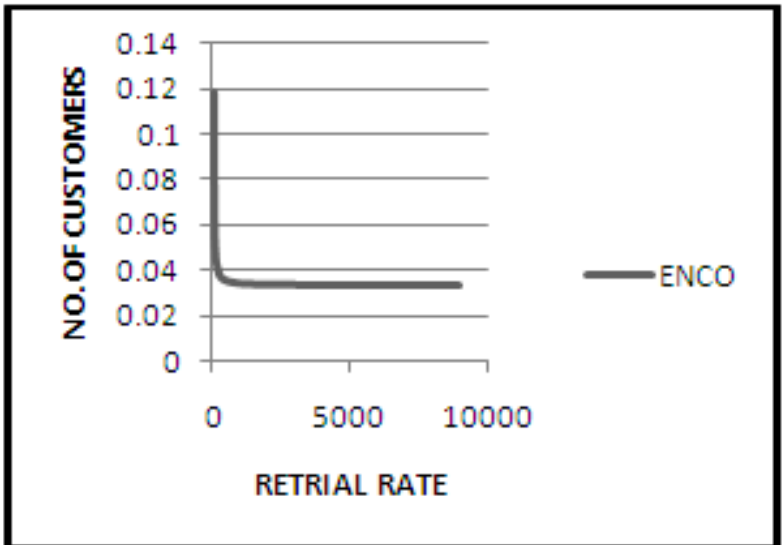

Figure 5.3 Expected Number of Customers in the Orbit When $\lambda=10 \mu=20 \mathrm{v}=20, \mathrm{~s}=3 \mathrm{~S}=6 \quad \lambda_{-1}=6$

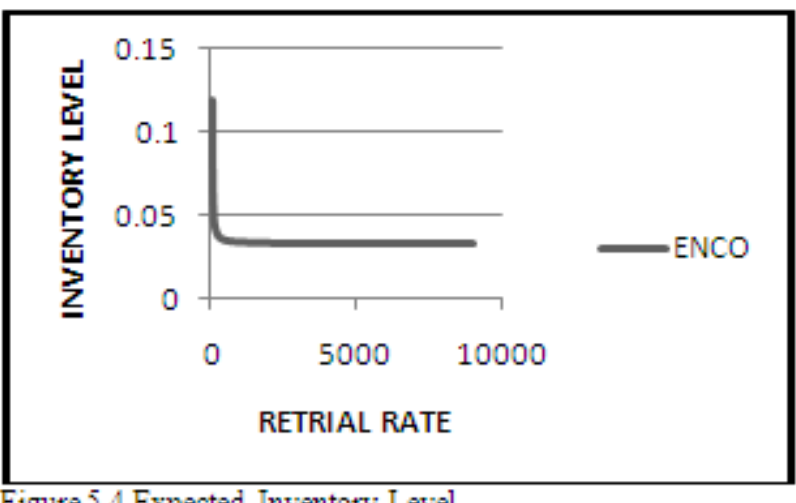

Figure 5.4 Expected Inventory Level

When $\lambda=10 \mu=20 \quad,=20, s=3 \quad \mathrm{~S}=6 \quad \lambda_{-1}=6$ 
Table 5.3 Variations in the

Negative arrival Rate $\lambda=10 \mu=20$

\begin{tabular}{|lccr|}
$\sigma=10$ & $s=3$ & $S=6 \quad v=10$ & \\
\hline$\lambda_{-1}$ & M & ENCO & EIL \\
\hline 2 & 21 & 0.2669 & 3.9345 \\
\hline 4 & 19 & 0.2299 & 3.9494 \\
\hline 6 & 11 & 0.1189 & 4.5003 \\
\hline
\end{tabular}

Table 5.4 Variations in the

Negative anival Rate $\lambda=10 \mu=20$

$\sigma=9000 \quad s=3 \quad S=6 \quad v=10$

\begin{tabular}{|llll|}
\hline$\lambda_{-1}$ & M & ENCO & EIL \\
\hline 2 & 21 & 0.1183 & 3.9129 \\
\hline 4 & 19 & 0.0992 & 3.9286 \\
\hline 6 & 17 & 0.0850 & 3.9414 \\
\hline
\end{tabular}

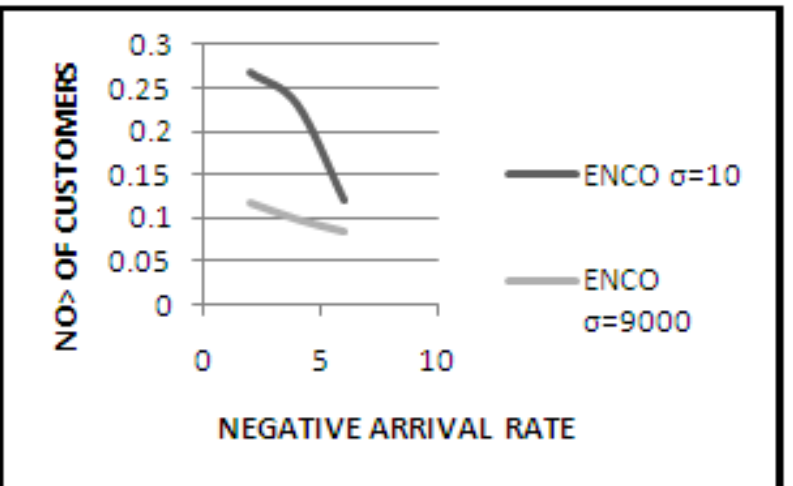

Figure 5.5 . Expected Number of Customers in the Orbit When $\lambda=10 \quad \mu=20 \quad s=3 \quad \mathrm{~S}=6, \quad y=10$

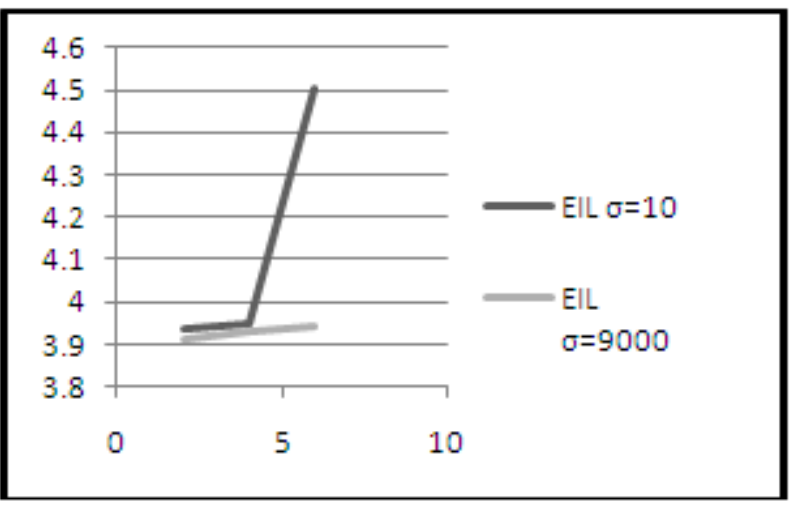

Figure 5,6 Expected Inventory Level

When $\lambda=10 \quad \mu=20 \quad s=3 \quad S=6 \quad y=10$

\section{CONCLUSIÓN}

In this paper a $(\mathrm{s}, \mathrm{S})$ inventory system with positive service time, lead time, retrial of customers and negative arrivals using matrix analytic method is studied and the following observations have been made.

From Tables 5.1, 5.2 and the given figures 5.1, 5.2, 5.3 and 5.4 it is seen that
- the ENCO decreases as the retrial rate increases

- the EIL decreases as the retrial rate increases

From Tables 5.3, 5.4 and the given figures 5.5, and 5.6 it is seen that

- the ENCO decreases as the negative arrival rate increases

- the EIL increases as the negative arrival rate increases

\section{REFERENCES}

[1] J. R. Artalejo and Antonio Gomez-Corral, Retrial Queueing Systems, Springer, 2008.

[2] Artalejo. J.R and A. Gomez corral (1999). On a single server queue with negative arrivals and repeated request, Journal of Applied Probability 36, pp 907-918.

[3] G. I. Falin, "A survey of retrial queues," Queueing Systems, vol. 7, no. 2, pp. 127-167, 1990.

[4] G. I. Falin and J. G. C. Templeton, Retrial Queues, Chapman \& Hall, London, UK, 1997.

[5] Gelenbe (1991). Product forms queueing networks with negative and positive customers, Journal of Applied Probability, 28, pp 656-663.

[6] P.G. Harrison and E. Pitel(1993). Sojourn times in single-server queue with negative customers, Journal of Applied Probability 30, pp 943-963.

[7] A. Krishnamoorthy and K. P. Jose, "Three Production Inventory Systems with Service, Loss and Retrial of Customers" Information and Management Sciences, Volume 19, Number 3, pp. 367-389, 2008

[8] J. R. Artalejo, A. Krishnamoorthy, and M. J. LopezHerrero, "Numerical analysis of $(s, S)$ inventory systems with repeated attempts," Annals of Operations Research, vol. 141, no. 1, pp. 67-83, 2006.

[9] A. Krishnamoorthy and M. E. Islam, " $(s, S)$ inventory system with postponed demands," Stochastic Analysis and Applications, vol. 22, no. 3, pp. 827-842, 2004.

[10] A. Krishnamoorthy and K. P. Jose, "An $(s, S)$ inventory system with positive lead-time, loss and retrial of customers ," Stochastic Modelling and Applications, vol. 8, no. 2, pp. 56-71, 2005.

[11] A. Krishnamoorthy and K. P. Jose "Comparison of Inventory Systems with Service, Positive Lead-Time, Loss, and Retrial of Customers" Journal of Applied Mathematics and Stochastic Analysis Volume 2007.

[12] A. Muthu Ganapathi Subramanian, G. Ayyappan, G. Sekar. "M/M/1 Retrial queueing system with negative arrival under non-preemptive priority service", Journal of Fundamental Sciences Vol 5, No 2 230-146, 2009

[13] P. V. Ushakumari, "On $(s, S)$ inventory system with random lead time and repeated demands," Journal of Applied Mathematics and Stochastic Analysis, vol. 2006 